\title{
Directional motion of dielectric droplets on polymer-coated conductor driven by electric corona discharge
}

Cite as: Appl. Phys. Lett. 114, 143701 (2019); https://doi.org/10.1063/1.5089296

Submitted: 18 January 2019. Accepted: 26 March 2019 . Published Online: 10 April 2019

Guoliang Li (D), Junheng Pan, Huai Zheng, Sheng Liu (D), and Jau Tang (iD)
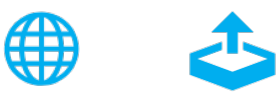

\section{ARTICLES YOU MAY BE INTERESTED IN}

Droplet actuation on superhydrophobic substrates via electric field gradients

Applied Physics Letters 114, 113702 (2019); https://doi.org/10.1063/1.5080241

Electrostatic capacitance-type acceleration of ions with an intense few-cycle laser pulse Applied Physics Letters 114, 144102 (2019); https://doi.org/10.1063/1.5088340

Atomic-layer-deposited $\mathrm{HfO}_{2} / \mathrm{Al}_{2} \mathrm{O}_{3}$ laminated dielectrics for bendable Si nanomembrane based MOS capacitors

Applied Physics Letters 114, 142903 (2019); https://doi.org/10.1063/1.5051626

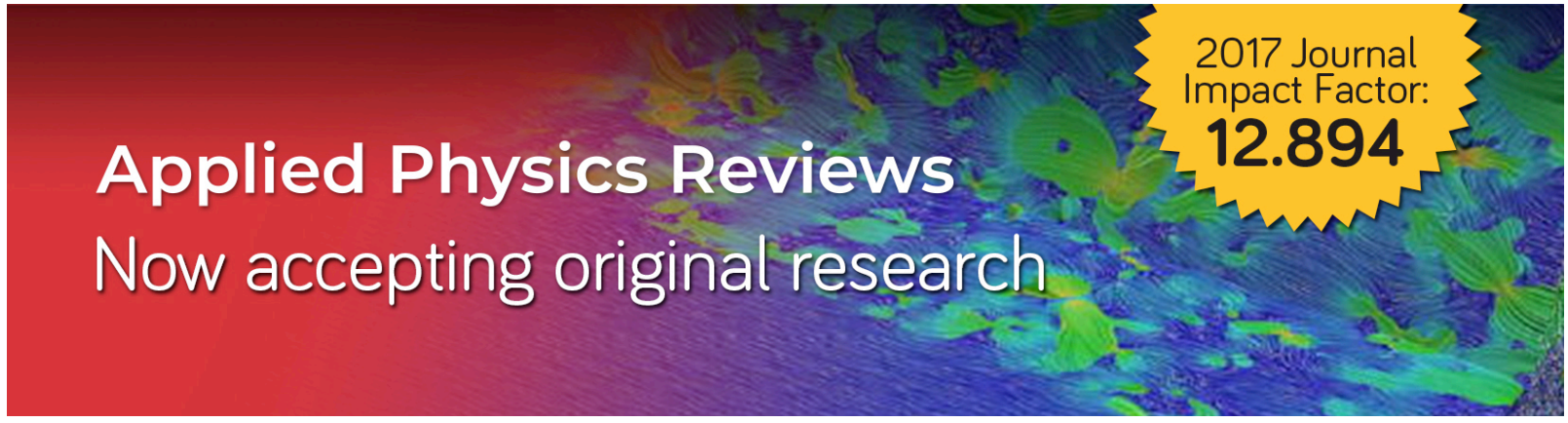




\title{
Directional motion of dielectric droplets on polymer-coated conductor driven by electric corona discharge
}

\author{
Cite as: Appl. Phys. Lett. 114, 143701 (2019); doi: 10.1063/1.5089296 \\ Submitted: 18 January 2019 • Accepted: 26 March 2019 • \\ Published Online: 10 April 2019
}
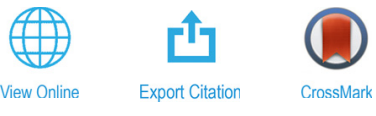

Guoliang Li, $^{1, a)}$ (D) Junheng Pan, ${ }^{1, a)}$ Huai Zheng, Sheng Liu, ${ }^{1,2, b)}$ (D) and Jau Tang ${ }^{2,3, b)}$ (D)

\begin{abstract}
AFFILIATIONS
${ }^{7}$ School of Power and Mechanical Engineering, Wuhan University, Wuhan, Hubei 430072, China

${ }^{2}$ Institute of Technological Sciences, Wuhan University, Wuhan, Hubei 430072, China

${ }^{3}$ Arthur Amos Noyes Laboratory of Chemical Physics, California Institute of Technology, Pasadena, California 91125, USA

a) Contributions: G. Li and J. Pan contributed equally to this work.

${ }^{b)}$ Authors to whom correspondence should be addressed: shengliu@whu.edu.ch and jautang@caltech.edu
\end{abstract}

\begin{abstract}
Achieving better control of liquid droplet movement is an important subject for academic research and industrial applications. In this study, we investigate the use of electric corona discharge to drive and control oil droplet movement. For a fully covered polymer-coated copper plate, all droplets on the surface were found to move outward in the same direction. Interestingly, for a polymer coating a hole directly beneath a needle biased with a high DC voltage, we observed the existence of a zone boundary at which all of the droplets within the boundary move inward, whereas droplets outside the boundary move outward. The boundary appeared to depend on the hole size and the voltage bias. Our model simulations reasonably agree well with the experimental measurements of these peculiar phenomena.
\end{abstract}

Published under license by AIP Publishing. https://doi.org/10.1063/1.5089296

In recent years, microfluidics has attracted considerable interest because of its promising applications in biology, ${ }^{1}$ chemistry, ${ }^{2}$ medicine, ${ }^{3}$ energy generation, ${ }^{4,5}$ heat management, ${ }^{6}$ and display technology. The ability to better control droplet dynamics is a critical component of microfluidics. Currently, different techniques have been developed as actuation methods in droplet control systems, including the use of air pressure, ${ }^{8}$ electrowetting, ${ }^{9-12}$ thermocapillary effects, ${ }^{13,14}$ dielectrophoresis, ${ }^{15,16}$ magnetohydrodynamics, ${ }^{17}$ structured surfaces, ${ }^{18}$ electrochemical gradients, photochemical effects, ${ }^{19}$ and other electrostatic techniques.

Phenomena that involve the direct conversion of electrical energy into kinetic energy are known as electro-hydrodynamics (EHD). ${ }^{21}$ Corona discharge refers to the phenomenon when the electric field near a conductor is sufficiently strong to ionize the dielectric surrounding it, but not sufficiently strong to cause an electrical breakdown or arcing between electrodes. ${ }^{21}$ One of the EHD effects is the electric wind caused by corona discharge. This phenomenon observed for several centuries has induced a variety of possible applications today and even showed a peculiar phenomenon, ${ }^{22}$ including flow enhancement, ${ }^{23}$ heat transfer enhancement, ${ }^{24}$ drying and evaporation, ${ }^{25}$ and fluid pumps. ${ }^{26}$ These applications and studies focused on a continuous fluid, and only a small number refers to the liquid droplet. $^{27-29}$

In this report, we describe some phenomena of dielectric droplet movement induced by electric corona discharge. Such a plasma-driven mechanism could provide a method to control the movement of dielectric droplets in potential industrial applications. Some experiments were designed to observe the action of the dielectric droplets in the presence of corona discharge.

In this experiment, as schematically illustrated in Fig. 1(a), a needle-to-plane configuration was adopted to produce a plasma when a high DC voltage was applied. A polyethylene terephthalate (PET) film of $0.1 \mathrm{~mm}$ thickness was coated on the electrode surface. A hole was carved out from the PET film to expose the underlying conductive substrate at the epicenter directly beneath the needle. We placed a drop of silicone oil $(\sim 0.2 \mu \mathrm{l})$ on the PET surface. As we abruptly connected the needle electrode to a DC power supply, which was preset at $9 \mathrm{kV}$, as shown in Fig. 1(b), a slightly flattened oil droplet instantly jumped up to form a round-shaped droplet and later slowly returned to the flat shape, even if the high voltage remained unchanged, while there was a horizontal movement on the surface (supplementary material Video 1). The direction of the droplet displacement was related to 
(a)

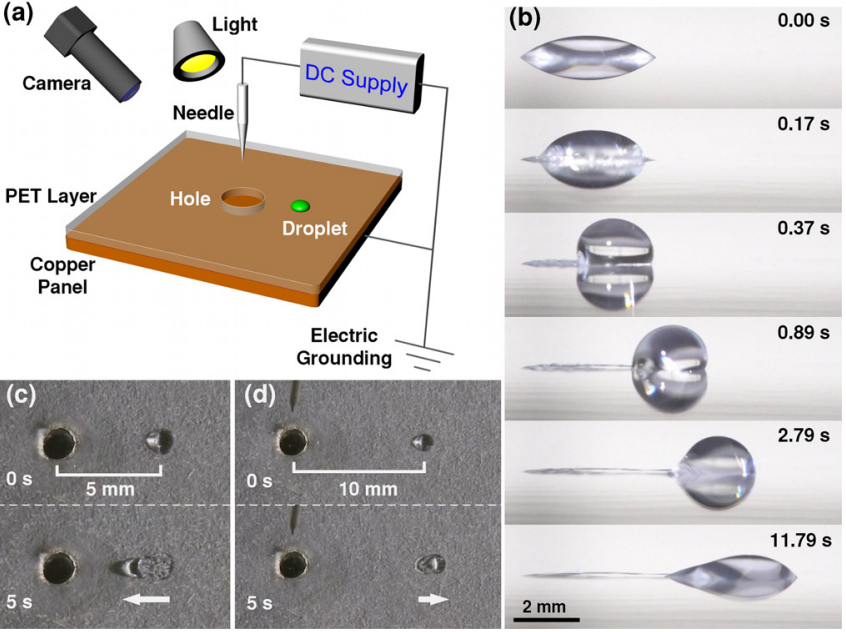

FIG. 1. (a) Schematic diagram for the experimental setup. (b) Horizontal angle view of the oil droplet and its surface reflection image. A flat droplet instantly jumped up to form a round-shaped droplet when a $9 \mathrm{kV}$ voltage was applied quickly, and the droplet slowly became flatter, even after the bias voltage was sustained. During the course of the experimental observation, the droplet moved sideways but with a trailing tail. The trailing tail is the liquid stuck to the substrate surface. (c) A droplet initially located $5 \mathrm{~mm}$ from the epicenter (top) moved toward the conductive hole after a $9 \mathrm{kV}$ voltage was suddenly applied (bottom). (d) A droplet initially $10 \mathrm{~mm}$ away from the epicenter (top) moved outward as a bias was suddenly applied (bottom).

the distance between the conductive hole and the droplet. Figure 1(c) shows that a droplet located at a $5 \mathrm{~mm}$ distance from the epicenter started to move toward the conductive hole when a voltage bias was applied to the electrode. In contrast, as shown in Fig. 1(d), for a droplet initially placed $10 \mathrm{~mm}$ from the conductive hole, a droplet moved away from the epicenter. These observations indicate that there is a zone boundary that divides the plane into an attractive region and a repulsive region.

Further experiments show that the direction of the droplet's displacement is relative to the distance between the droplet and the conductive hole. As shown in Figs. 2(a)-2(c) (supplementary material Video 2), several droplets were placed in a spiral arrangement. These droplets were located as close to the conductive hole as possible, and the farthest one was $7 \mathrm{~mm}$ from the epicenter [Fig. 2(a)]. When a 9$\mathrm{kV}$ voltage was applied, every droplet moved toward the conductive hole [Figs. 2(b) and 2(c)], and the direction of every droplet was radial inward. The experiments shown in Figs. 2(d)-2(f) (supplementary material Video 3) exhibit an opposite appearance. Several droplets were placed in a spiral arrangement, similar to Fig. 2(a), but at a larger distance from the conductive hole. The nearest droplet was $9 \mathrm{~mm}$ from the epicenter [Fig. 2(d)]. When a $9 \mathrm{kV}$ voltage was applied, every droplet moved outward from the conductive hole [Figs. 2(e) and 2(f)], and the direction of every droplet was radial outward. Experiments shown in Figs. 2(g)-2(i) (supplementary material Video 4) confirmed the phenomenon. Droplets were also placed in a spiral arrangement but with a larger range than the above two experiments [Fig. 2(g)]. When a $9-\mathrm{kV}$ voltage was applied, all of the droplets moved, though in a different direction: the droplets located near the epicenter moved inward, similar to Fig. 2(a), while the droplets located farther out moved out, similar to Fig. 2(d), and if a droplet had a distance from

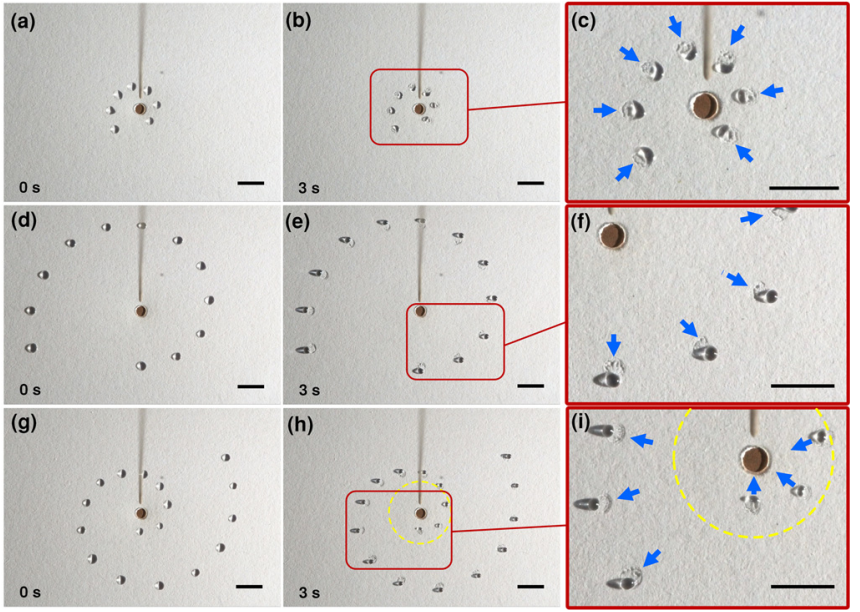

FIG. 2. (a)-(c) Vertical view of droplets placed in a spiral arrangement and close to the epicenter. Every droplet moved toward the conductive hole after a $9 \mathrm{kV}$ voltage bias was applied. (a) Droplets in their initial position. (b) Droplets moving toward the conductive hole. (c) Partial enlargement of the image (b). (d)-(f) Vertical view of several droplets that were placed in a spiral arrangement, but sighted farther from the epicenter. Every droplet moved outward from the conductive hole after the same voltage was applied. (d) Droplets in their initial position. (e) Droplets moved outward from the conductive hole. (f) Partial enlargement of the image (e). (g)-(i) The droplets were placed in a spiral arrangement and in a larger span range. Droplets within the boundary (yellow dotted circle) appeared to move inward, whereas those outside the boundary moved outward. (g) Initial position. (h) Voltage was applied. (i) Partial enlargement of the image (h). The bar represents $5 \mathrm{~mm}$ in length in all the figures.

the epicenter that was not too close and not too far, it would stay still and did not show an obvious displacement [Figs. 2(h) and 2(i)].

This phenomenon implies that there is a border, which is schematically illustrated by a dashed line in Fig. 3(a), dividing the plane surface to the two domains. All droplets located inside the ring boundary move toward the conductive hole; conversely, all of the droplets located outside the ring boundary move away from the conductive hole. Figure 3(b) shows a record of the distance between the droplets and the epicenter for 7 droplets, which were distributed near the ring boundary. Therefore, we could define the ring border as a threshold, dividing the plane surface into two domains. We studied the range of the threshold as a function of the radius of the conductive hole and the voltage bias. Figure 3(c) shows the experimental results of the relationship between the radius of the threshold and the radius of the conductive hole. This figure reveals a linear relationship with a fitted slope of 1.13. This result indicates that when the radius of the conductive hole increases, the radius of the threshold increases proportionally. Figure 3(d) shows the experimental results of the relationship between the radius of the threshold and the voltage with a $3 \mathrm{~mm}$ radius of the conductive hole. The results indicate a linear relationship with a fitted slope of $0.32 \mathrm{~mm} / \mathrm{kV}$.

We analyzed the velocity of the droplets at different positions. In the experiment with a conductive hole diameter of $2 \mathrm{~mm}$, we chose three droplets within the threshold radius which were located at 3.8, 5.0 , and $6.3 \mathrm{~mm}$, respectively, from the center and three droplets outside the threshold radius which were located at 11.0, 14.5, and $28.7 \mathrm{~mm}$, respectively, from the center. The velocity of each droplet 
(a)

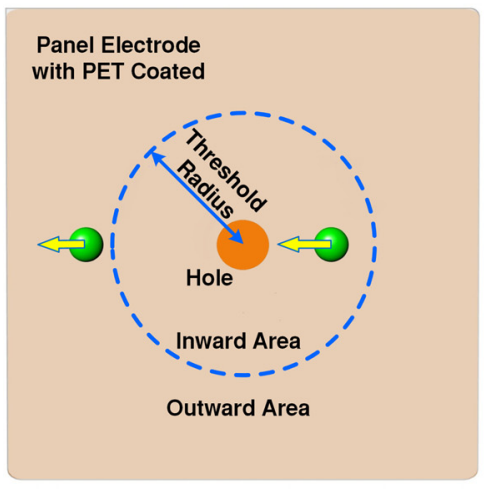

(c)

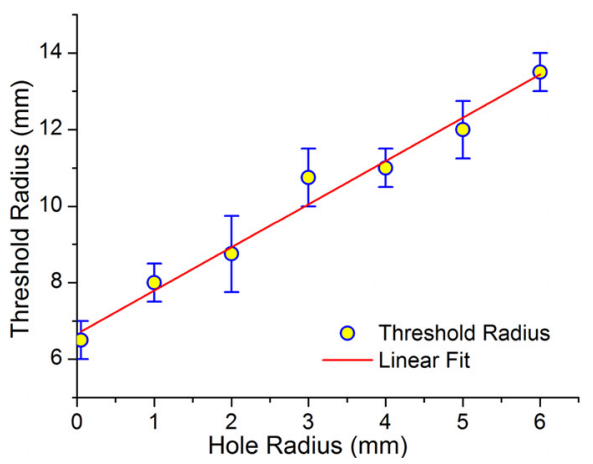

(b)

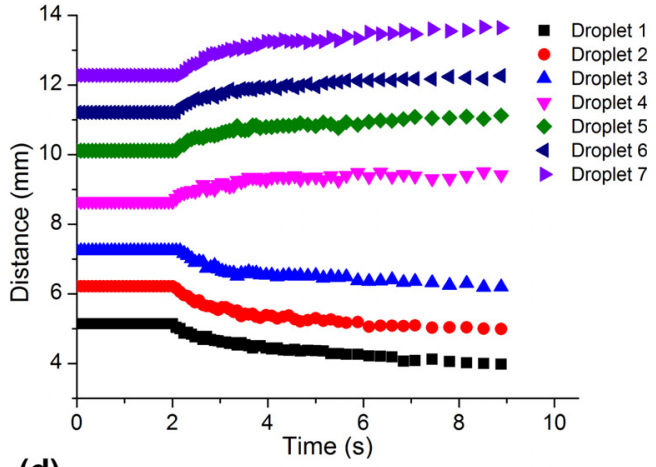

(d)

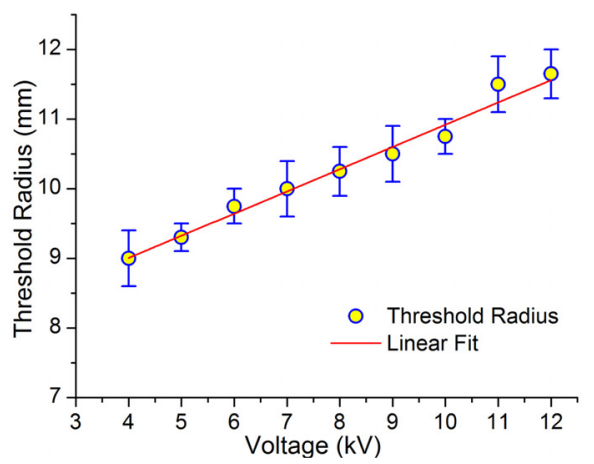

FIG. 3. (a) Schematic illustration of the boundary (blue dotted circle) which divides the inward area from the outward area for the droplet movement. (b) The time evolution for the position of seven droplets which were initially sighted near and far from the center. The voltage was applied for $2 \mathrm{~s}$. The error of each point is $0.2 \mathrm{~mm}$. (c) Experimental data showing the corresponding threshold radius vs the conductive hole radius at $9 \mathrm{kV}$, with a fitted slope of 1.13. (d) The voltage dependence of the threshold radius with a conductive hole radius of $3 \mathrm{~mm}$ with a fitted slope of $0.32 \mathrm{~mm} / \mathrm{kV}$. can be obtained by fitting the experimental record of droplet position evolution with time. These experimental data could be fitted well by a bi-exponential function. Then, the velocity curve is obtained from the fitting function. As shown in Fig. 4(a), the droplets have a faster startup speed and then gradually slow down. Within the threshold radius, the droplet has a faster start-up speed when it is located closer to the conductive hole. Outside the threshold radius, the fastest start-up speed of the droplet appeared in a range with a certain distance from the threshold radius, near or farther, the start-up speed would be slower.

We also considered the influence of some experimental conditions on the motion of droplets. Figure 4(b) shows the extent of droplet deformation on PET layers of different thicknesses. We used the ratio of droplet height change to the droplet initial diameter as the extent of droplet deformation (Fig. S1). The arrows in Fig. 4(b) indicate the moment when the greatest extent of droplet deformation appeared on different thickness PET layers. A thicker polymer layer causes droplet deformation to become weaker and tardier. But we did not find any significant effect of polymer thickness on the threshold radius. Figure 4(c) shows that the displacement of droplets on different roughness PET layers indicates that the droplet moves slowly on a rougher polymer surface. However, the threshold radius was not found to be affected by using PET layers with different roughness values. The topography and the roughness of each surface are shown in Fig. S2. We also used droplets with different viscosities in our experiments. Figure 4(d) shows that the droplets of low viscosity have a greater displacement than that of a droplet with high viscosity. And, the viscosity of the droplet does not affect the threshold radius.
To elucidate the causes of the zone boundary for the opposite movement direction, we proposed a model and conducted model simulations. Using COMSOL software (COMSOL Inc.), we calculated the charge distribution on the PET surface and the electric field in air due to high DC voltage discharge. The movement of the oil droplets is the result of the charged oil molecules interacting via Coulomb forces with other charges on the surface and with the electric field generated by the sharp needle with a high DC voltage and a grounding conductor substrate. The fundamental equations for calculation of the electric field intensity and the electric potential are the following: ${ }^{30,31}$

$$
\begin{gathered}
\nabla \times \mathrm{J}=-\frac{\partial \rho}{\partial t}, \\
J=\left(\sigma+\varepsilon_{0} \varepsilon_{r} \frac{\partial}{\partial t}\right) E+J_{e}, \\
E=-\nabla V,
\end{gathered}
$$

where J denotes the current density, $\rho$ is the electric charge density, $t$ is the time, $\sigma$ is the electrical conductivity $\varepsilon_{0}$ is the permittivity of vacuum, $\varepsilon_{r}$ is the permittivity of PET, $E$ is the electric field intensity, $J_{e}$ is the externally generated current, $V$ is the electric potential, and $\nabla$ is the gradient symbol.

Figure 5(a) shows the horizontal component for the calculated electric field on the PET film surface. The positive values of electric field intensity indicate the radial outward direction and the negative values indicate the radial inward direction. The horizontal component of the electric field intensity determined the direction of Coulomb force applied on the droplet and, as a result, determined the direction 
(a)

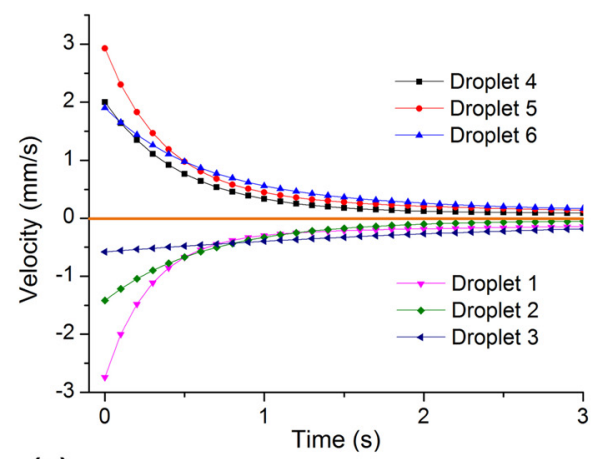

(c)

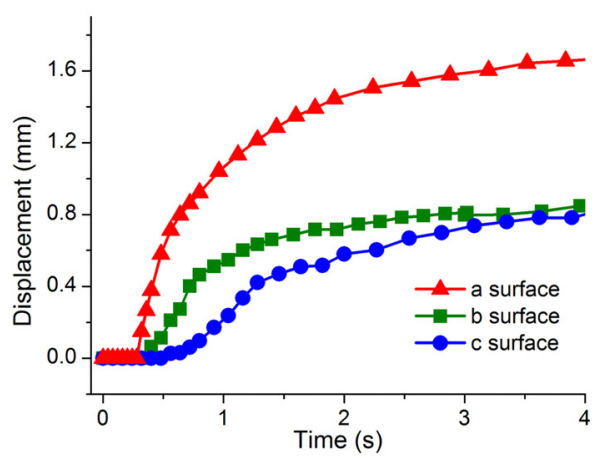

(a)

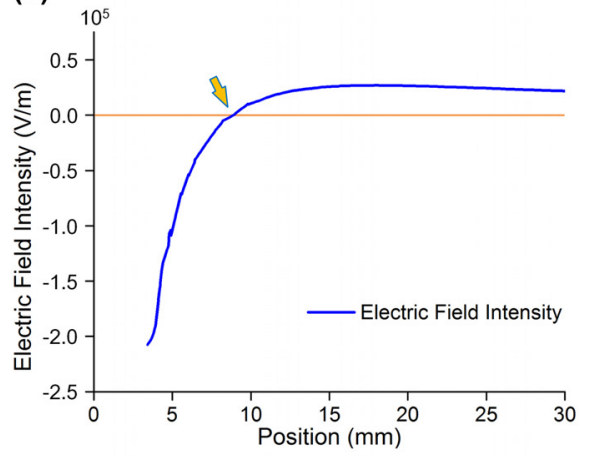

(c)

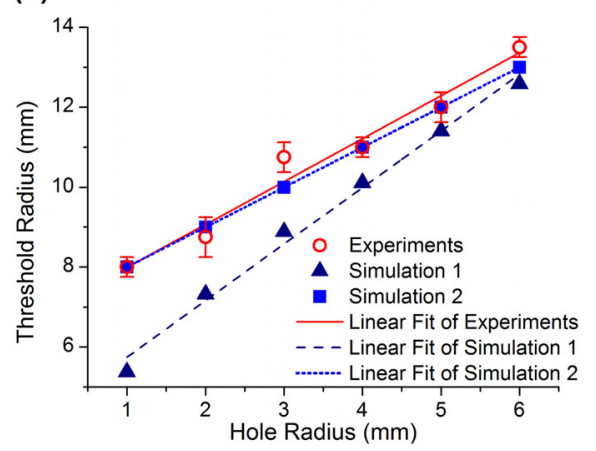

(b)

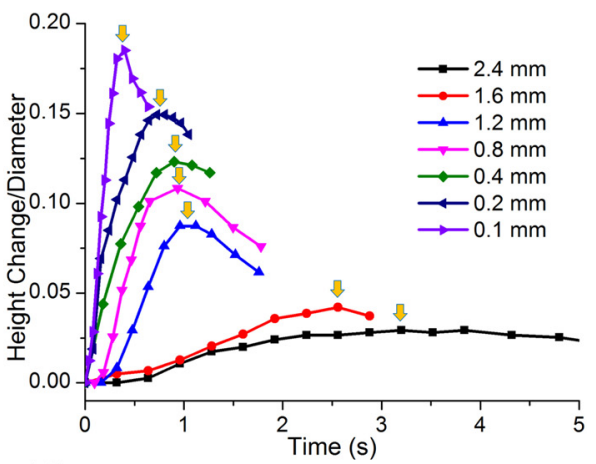

(d)

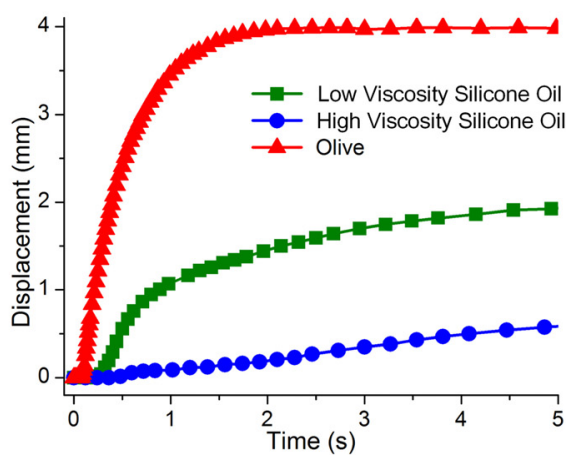

(b)

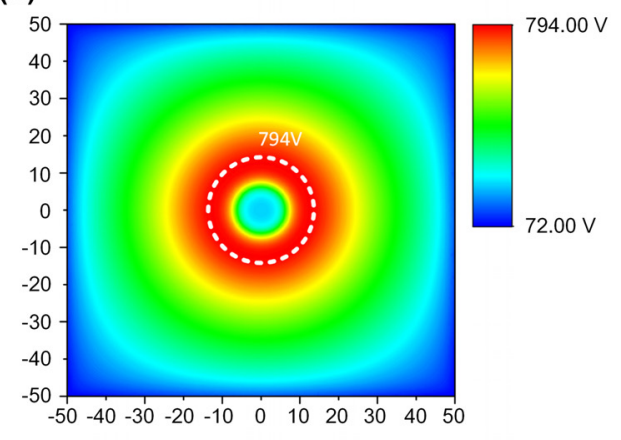

(d)

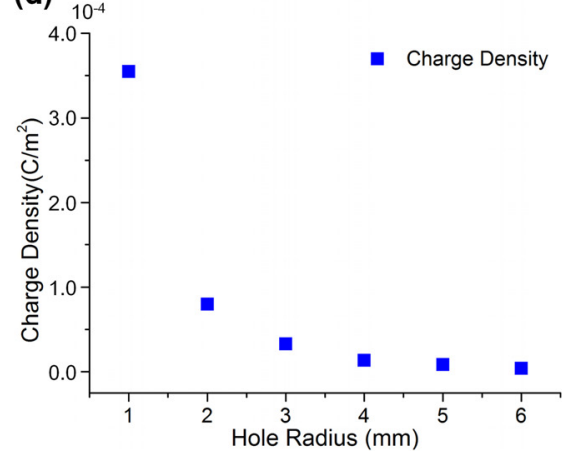

FIG. 4. (a) The time evolution for the velocity of six droplets which were initially located at 3.8, 5.0,6.3, 11.0, 14.5, and $28.7 \mathrm{~mm}$, respectively, from the center. The velocity was obtained by deriving the fitting function of the relationship between the droplets position and the time. (b) Experimental data showing the time evolution for the extent of droplet deformation (ratio of droplet height change to droplet initial diameter) at different PET layer thicknesses. The arrow on each curve indicates the moment at which the droplet deformation is greatest. Droplets deformed more slowly and slightly on a thicker PET layer. (c) The time evolution for the position of droplets on a PET layer with different roughness surfaces. (d) The time dependence of the position of different droplets, which are low viscosity silicone oil with 1000 mpa s viscosity, high viscosity silicone oil with 25000 mpa s viscosity and olive oil with $81 \mathrm{mpa} s$ viscosity.
FIG. 5. Model simulation results. (a) The horizontal component of the calculated electric field on the PET film, indicating the existence of a curve crossing with the $x$-axis indicated by an arrow to separate the regimes of outward and inward motion. (b) Electric potential simulation on the PET film surface. The position which appears maximum coincided with the position with a zero-horizontal component of the electric field. (c) The dependence of the threshold radius on the conductive hole radius. (d) Charge density was added to fit the experiment. 
of droplet displacement. The position represented by the 0 value is the threshold. And, this curve reveals that the horizontal component of electric field intensity is larger when the position is closer to the center in the inward area. However, as the distance from the center increases, the horizontal component of the electric field first becomes stronger and then becomes weaker. The fit to the velocity of droplets distributed at different positions appears reasonably well by the experiments shown in Fig. 4(a). Electric potential simulation on the PET film surface is shown in Fig. 5(b). The position which appears maximum coincided with the threshold. Figure 5(c) shows the relationship between the threshold radius and the conductive hole radius. The triangle icons represent the direct results by simulation. There is a non-negligible gap between the simulated data and the experimental data. We consider that this effect may be caused by ignoring the PET surface charge caused by corona which COMSOL did not add in its default calculation. We added an even charge distribution to approximately simulate the charge produced by corona on the PET surface and gave a more suitable simulation result represented by the square icon. The charge density set, as shown in Fig. 5(d), fits the experiment data well. The simulated results appear to be in good agreement with our experimental observation.

In this report on the control of droplet movement induced by electric corona discharge, we describe some interesting phenomena for the existence of a zone boundary that dictates whether the droplet moves inward or outward. We measured the dependence of the zone size on the hole size and the DC voltage bias. We proposed a model and conducted simulations to explain such peculiar behavior, and we obtained reasonably good agreement between the experiment and the theory. This study elucidates a mechanism with two major driving forces for the droplet movement. One dominant driving force is due to the Coulomb interactions of the droplet with the electric field that were established by the biased sharp needle and the polymer-coated electrode. This electric field, based on our simulations, explains most of the experimental features. To achieve a better quantitative agreement between the experimental curve and the simulated curve, a minor correction for the Coulomb force, due to the surface electric field induced by the positively charges on the polymer, resided on the polymer overcoat. By including such a factor of Coulomb repulsion on the surface, the zone boundary would appear slightly larger. With that approach, we obtained a reasonable quantitative fit. With a better understanding of the physical mechanism of electric corona discharge and the relevant Coulomb interactions, one could develop microfluidic techniques to more precisely control fluid movement in potential industrial applications.
See supplementary material for the experiment design, simulation, error analysis, and supplementary videos.

This work was financially supported by the National Natural Science Foundation of China (Northwest Key Scientific Facility, Grant No. 51727901)

\section{REFERENCES}

${ }^{1}$ E. K. Sackmann, A. L. Fulton, and D. J. Beebe, Nature 507(7491), 181 (2014).

${ }^{2}$ S. Mashaghi, A. Abbaspourrad, D. A. Weitz, and A. M. van Oijen, TrACTrends Anal. Chem. 82, 118 (2016).

${ }^{3}$ B. Harink, S. Le Gac, R. Truckenmueller, C. van Blitterswijk, and P. Habibovic, Lab Chip 13(18), 3512 (2013).

${ }^{4}$ M. Uhlen and H. A. Svahn, Lab Chip 11(20), 3389 (2011).

${ }^{5}$ E. R. Choban, L. J. Markoski, A. Wieckowski, and P. J. A. Kenis, J. Power Sources 128(1), 54 (2004).

${ }^{6}$ G. Maltezos, A. Rajagopal, and A. Scherer, Appl. Phys. Lett. 89(7), 074107 (2006).

${ }^{7}$ R. A. Hayes and B. J. Feenstra, Nature 425(6956), 383 (2003).

${ }^{8}$ K. Handique, D. T. Burke, C. H. Mastrangelo, and M. A. Burns, Anal. Chem. 73(8), 1831 (2001).

${ }^{9}$ F. Mugele and J. C. Baret, J. Phys.-Condens. Matter 17(28), R705 (2005).

${ }^{10}$ M. G. Pollack, A. D. Shenderov, and R. B. Fair, Lab Chip 2(2), 96 (2002).

${ }^{11}$ M. G. Pollack, R. B. Fair, and A. D. Shenderov, Appl. Phys. Lett. 77(11), 1725 (2000).

${ }^{12}$ E. Colgate and H. Matsumoto, J. Vac. Sci. Technol., A 8(4), 3625 (1990).

${ }^{13}$ T. S. Sammarco and M. A. Burns, AIChE J. 45(2), 350 (1999).

${ }^{14}$ A. A. Darhuber, J. P. Valentino, S. M. Troian, and S. Wagner, J. Microelectromech. Syst. 12(6), 873 (2003).

${ }^{15}$ J. S. Batchelder, Rev. Sci. Instrum. 54(3), 300 (1983).

${ }^{16}$ T. B. Jones, M. Gunji, M. Washizu, and M. J. Feldman, J. Appl. Phys. 89(2), 1441 (2001).

${ }^{17}$ A. Ahmadi, C. M. Mcdermid, and L. Markley, Appl. Phys. Lett. 108(1), 014101 (2016).

${ }^{18}$ O. Sandre, L. Gorre-Talini, A. Ajdari, J. Prost, and P. Silberzan, Phys. Rev. E 60(3), 2964 (1999).

${ }^{19}$ M. Nakagawa and K. Ichimura, Mol. Cryst. Liq. Cryst. Sci. Technol., 345, 599 (2000).

${ }^{20}$ J. Lee and C. J. Kim, J. Microelectromech. Syst. 9(2), 171 (2000).

${ }^{21}$ E. D. Fylladitakis, M. P. Theodoridis, and A. X. Moronis, IEEE Trans. Plasma Sci. 42(2), 358 (2014).

${ }^{22}$ G. Li, R. Hu, J. Tang, H. Zheng, and S. Liu, Sci. Rep. 8, 17813 (2018).

${ }^{23}$ E. Moreau, L. Leger, and G. Touchard, J. Electrost. 64(3-4), 215 (2006).

${ }^{24}$ P. H. G. Allen and T. G. Karayiannis, Heat Recovery Syst. CHP 15(5), 389 (1995).

${ }^{25}$ F. C. Lai and R. K. Sharma, J. Electrost. 63(3-4), 223 (2005).

${ }^{26}$ S. F. Bart, L. S. Tavrow, M. Mehregany, and J. H. Lang, Sens. Actuators, A 21(1-3), 193 (1990).

${ }^{27}$ Y. Higashiyama, S. Yanase, and T. Sugimoto, J. Electrost. 55(3-4), 351 (2002).

${ }^{28}$ Y. Zhu, M. Otsubo, and C. Honda, Surf. Coat. Technol. 201(9-11), 5541 (2007).

${ }^{29}$ S. Zhou, H. Zheng, G. Li, J. Liu, and S. Liu, Appl. Surf. Sci. 436, 839 (2018).

${ }^{30} \mathrm{R}$. Bansal, Handbook of Engineering Electromagnetics (CRC Press, 2014).

${ }^{31}$ J. Jin, The Finite Element Method in Electromagnetics (Wiley-IEEE Press, 2002). 